\title{
Relación entre bioética e investigación en ciencias de la vida en Argentina: estudio descriptivo a escala nacional
}

\author{
Paula Sánchez Thevenet ${ }^{1}$, Carmen Javaloyes Del Río ${ }^{2}$
}

Resumen: Los objetivos de este estudio son explorar y actualizar la relación entre bioética e investigación en ciencias de la vida en Argentina, a escala nacional. Para ello se realizó un estudio descriptivo, transversal, de análisis de contenido y corte bibliométrico, sobre productos públicos observables de carácter nacional. Se encontraron elementos propios de la bioética en las normas nacionales relacionadas con la investigación científica en este campo de las ciencias. Se evidenció que la solicitud de requerimientos de ética en la investigación científica para la publicación de los trabajos en dichas ciencias, es materia a optimizar en el país. Se constató el aporte de autores argentinos a la producción bibliográfica en la disciplina, desde 1988. Los resultados indican situaciones que contribuirían a una consolidación de la disciplina bioética en relación con las ciencias de vida en Argentina.

Palabras clave: bioética, ciencia, vida, Argentina

\section{Relationship between bioethics and life sciences research in Argentina: Descriptive study at national level}

\begin{abstract}
The aim of this study is to explore and update the relationship between bioethics and life sciences research in Argentina at national level. For this a descriptive, transversal, content analysis and bibliometric section study was carried out about observable public products of national character. Bioethical elements were found in national norms related to scientific research in this science field. It was evident that the ethical requirements asked in scientific research for the publication of studies in these sciences needs optimization. The contribution of Argentinean authors to bibliography production in the discipline was established since 1988. Results indicate situations that contribute to a consolidation of bioethics discipline in relation to life sciences in Argentina.
\end{abstract}

Key words: bioethics, science, life, Argentina

\section{Relaçáo entre a bioética e a investigação nas ciências da vida na Argentina: estudo descritivo em escala nacional}

Resumo: Os objetivos deste estudo são explorar e atualizar a relação entre a bioética e a investigação em ciências da vida na Argentina em escala nacional. Para isso se realizou um estudo descritivo, transversal, de análise de conteúdo e corte bibliométrico sobre produtos públicos observáveis de caráter nacional. Foram encontrados elementos próprios da bioética nas normas nacionais relacionadas com a investigação científica neste campo das ciências. Foi evidenciado que a solicitação de requerimentos de ética na investigação científica para a publicação dos trabalhos nas ditas ciências é matéria a ser otimizada no país. Foi constatado o aporte de autores argentinos na produção bibliográfica da disciplina, desde 1988. Os resultados indicam situações que contribuiriam para uma consolidação da disciplina bioética em relação às ciências da vida na Argentina.

Palavras-chave: bioética, ciência, vida, Argentina

\footnotetext{
${ }^{1}$ Doctora en Bioquímica, Máster en Bioética. Investigador del Centro Regional de Investigación y Desarrollo Científico Tecnológico (CRIDECIT), Facultad de Ciencias Naturales, Universidad Nacional de la Patagonia San Juan Bosco (UNPSJB), Chubut, Argentina

Correspondencia: paula_sanchezthevenet@yahoo.com.ar

${ }^{2}$ Licenciada en Farmacia, Máster en Bioética, Universidad Católica de Valencia, España
} 


\section{Introducción}

La relación entre ética y ciencia está en constante evolución, y surge en ella, como un tema central de análisis, si todo lo técnicamente posible es éticamente admisible $(1,2)$.

La relación ciencia/ética ha transitado por distintas etapas, destacándose tres momentos claves de la misma. Un primer momento ocurrido en la antigua Grecia (350-450 a.C), en el que ciencia y ética no designaban dominios separados que hubiera que relacionar posteriormente(3). Luego, a partir del siglo XVIII - y manteniendo su vigencia hasta las últimas décadas del siglo $\mathrm{XX}$-, se da otro momento singular, correspondiente al desarrollo de la ciencia moderna. Durante ese período transcurre la etapa de la "neutralidad científica", caracterizada por la idea de una ciencia exenta de juicios éticos. Actualmente tiene lugar una nueva fase, asociada a la ciencia posmoderna. En este tiempo, el desafío que supone procurar un progreso científico acorde con el bien de la humanidad y del ecosistema —entendiéndose el bien como una ley moral(4) — brinda un escenario de crisis de aquella neutralidad científica. La idea de ciencia libre de valoraciones éticas empieza a verse debatida no sólo desde la propia comunidad científica, si no también desde la sociedad en general(2).

En ese contexto y en particular en países emergentes o empobrecidos, la investigación biomédica, biotecnológica y ambiental supone una serie de circunstancias especiales, debido a la carencia total o parcial de un marco jurídico y ético que proteja a los sujetos de investigación(5) y al ambiente natural. Para conocer dicho marco en una sociedad determinada resulta útil la observación de productos sociales que identifican discursos compartidos por dicha sociedad: los productos públicos observables(6). Ejemplos de estos productos son, entre otros, el ordenamiento jurídico relativo a la praxis científica, los reglamentos de publicación de las revistas especializadas y el aporte a la producción bibliográfica internacional, desde un país o región, en la disciplina en cuestión.
La bioética llega a la Argentina a instancias de J.A. Mainetti, quien crea la Fundación que lleva su nombre en 1982. Luego, en la década de los '90, la disciplina se va canalizando a través de los comités de ética clínica y surge como principal instrumento relacionado con la ética en el contexto de la investigaciones médicas, el consentimiento informado(7). Hacia 2003, Outomuro(8) consideró que la bioética en el país transcurría por tres etapas de sustanciación disciplinar, las cuales se daban en forma conjunta: la etapa emotiva, la reconstructiva y la de consolidación. En otro trabajo, dicha autora señala que, aunque existía una creciente toma de conciencia sobre la inclusión de la reflexión ética en el ámbito sanitario, no se evidenciaba en el campo de la investigación científica una situación semejante(9). En cuanto al marco jurídico referido a la ciencia en este país, un estudio de $\operatorname{EULABOR}(10)^{3}$ pone en evidencia, al año 2005, la falta de un sistema nacional de revisión ética de la investigación biomédica, con una autoridad de aplicación que tenga facultades de registro, aprobación y control de los estudios. Los últimos datos encontrados sobre bioética y ciencia en Argentina se refieren a la vulnerabilidad de los sujetos participantes en una investigación y al consentimiento informado $(11,12)$.

En función de lo señalado, los objetivos de este trabajo son explorar y actualizar el estado de la relación entre bioética e investigación en ciencias de la vida en Argentina, a escala nacional.

\section{Metodología}

Diseño del estudio: descriptivo, transversal, de análisis de contenido y corte bibliométrico(13).

\section{Lugar de estudio: República Argentina.}

La República Argentina está situada en el extremo sureste de América, siendo el segundo país más extenso de América del Sur. Su territorio está dividido en 23 provincias y una ciudad autónoma, la Ciudad Autónoma de Buenos Aires, capital de la República y sede del gobierno nacional. Este

\footnotetext{
3 European and Latin American Systems of Ethics Regulation of Biomedical Research.
} 
país tiene una población estimada de 40 millones de habitantes 4 . En la actualidad, Argentina está considerada como un mercado emergente y es la tercera potencia en materia económica de América Latina.

El sistema de gobierno del país se divide en tres poderes, ejecutivo, legislativo y judicial. El poder ejecutivo es desempeñado por el presidente de la Nación; el legislativo, por el Congreso de la $\mathrm{Na}$ ción, integrado por dos cámaras, la Honorable Cámara de Diputados y la Honorable Cámara de Senadores. Dicho Congreso está a cargo de la formación y sanción de las leyes nacionales y los códigos legales civil, penal, comercial, laboral, aeronáutico y de minería, entre otros. El poder judicial es ejercido por la Corte Suprema de Justicia y por los jueces y tribunales de las diversas instancias y jurisdicciones.

La legislación en Argentina tiene al menos tres niveles, nacional, provincial y municipal. La Constitución Nacional es la ley suprema de la Nación ${ }^{5}$, primaria y fundante del orden jurídico, por lo que las demás normas deben adecuarse a ella. Por debajo de la Constitución rigen, en orden de prelación normativa, las leyes nacionales, los decretos del poder ejecutivo, el resto de las normas nacionales - resto de los decretos, disposiciones, resoluciones-, las constituciones provinciales, el resto de las normas provinciales, las constituciones o cartas municipales y el resto de las normativas municipales. Las provincias pueden sancionar leyes sobre cuestiones no federales, si bien las principales leyes comunes — civiles, comerciales, penales, laborales, de seguridad social y de minería- están reservadas al Congreso Nacional.

\section{Objetos de estudio}

Se exploraron y analizaron los siguientes productos públicos observables de carácter nacional: (i) normas nacionales vinculadas a la investigación científica en ciencias de la vida, (ii) reglamentos de revistas argentinas relacionadas con dichas ciencias y (iii) producción bibliográfica sobre bioética

\footnotetext{
${ }^{4}$ Información obtenida del sitio web oficial de Argentina: http:// www.argentina.gov.ar/ . Fecha de acceso 17/08/2009.

${ }^{5}$ Junto con los tratados internacionales con jerarquía constitucional.
}

de autoría total o parcial con filiación argentina. Se adoptó la clasificación de UNESCO para identificar los campos de aquellas ciencias(14).

Las normas de alcance nacional ${ }^{6}$ analizadas fueron las sancionadas hasta el período parlamentario $2008^{7}$. Sobre dichas normas se evaluó la presencia en su contenido de elementos de bioética y se registró el año de sanción y el tipo de norma. Las regulaciones sobre investigación farmacológica se obtuvieron del sitio web de la Administración Nacional de Medicamentos, Alimentos y Tecnología Médica (ANMAT) ${ }^{8}$ : http://www. anmat.gov.ar/. El resto de las normas nacionales se obtuvo de archivos propios y de los sitios web especializados y oficiales de Argentina: Sistema Argentino de Informática Jurídica del Ministerio de Justicia, Seguridad y Derechos Humanos de la Nación —http://www.saij.jus.gov.ar/—y Centro de Documentación e Información del Ministerio de Economía y Finanzas de la República Argentina área información legislativa — http://infoleg. mecon.gov.ar/. Además, se consultó a la Honorable Cámara de Senadores de la Nación y a la Honorable Cámara de Diputados de la Nación sobre la legislación nacional vigente y sobre proyectos de ley en materia de ética de la investigación en ciencias de la vida.

Sobre los reglamentos de revistas relacionadas con ciencias de la vida, se analizaron los requerimientos de ética para la publicación de trabajos. Se registró, a su vez, el año de actualización del reglamento y carácter del comité evaluador de la revista. La muestra estudiada consistió en 21 reglamentos obtenidos de (i) 14 revistas seleccionadas a partir de las 32 obrantes en las áreas de ciencias biológicas y de ciencias de la salud del Núcleo Básico de Revistas del Centro Argentino de Información Científica y Tecnológica (CAICYT), al 02/10/2008, y (ii) 7 revistas pertenecientes a

\footnotetext{
${ }^{6}$ No se analizó para el presente estudio la Constitución Nacional.

7 Este último período comprende desde el 1/3/2008 hasta el 28/02/2009

${ }^{8}$ ANMAT es un organismo de la Administración Pública Nacional de Argentina que tiene todo el territorio nacional como jurisdicción y depende técnica y científicamente de la Secretaria de Políticas, Regulación e Institutos del Ministerio de Salud de la Nación. En materia de investigación, ANMAT regula sobre Buenas Prácticas de Investigación en Farmacología Clínica, Ensayos Clínicos e Investigación Clínica con Productos de Tecnología Médica.
} 
colectivos científico/profesionales o editadas en universidades nacionales, obtenidas a marzo de 2009.
La producción bibliográfica sobre bioética y de autoría total o parcial con filiación argentina se estudió a partir de la producción indizada en las

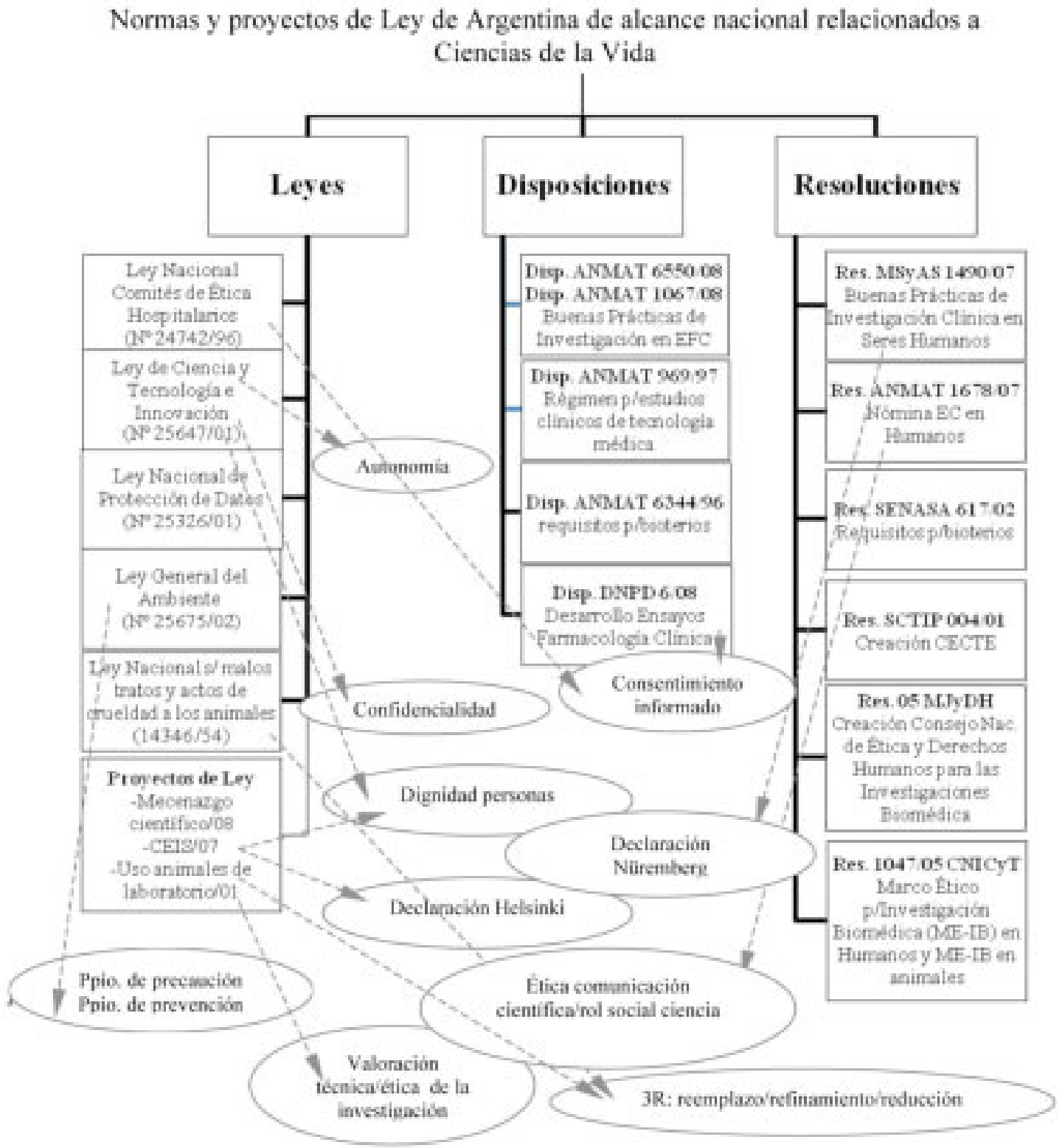

Diagrama 1. Normas nacionales y proyectos de ley relacionados con la investigación en ciencias de la vida y presencia de elementos de la bioética. Argentina, compilación hasta marzo de 2009.

Siglas: CECTE: Comité Nacional de Ética en la Ciencia y la Tecnología; MSyAS: Ministerio de Salud y Acción Social; DNPD: Dirección Nacional de Protección de Datos; CEIS: Comités de Ética de la Investigación en Salud; CNICyT: Consejo Nacional de Investigación en Ciencia y Tecnología; SCTIP: Secretaría de Ciencia, Tecnología e Innovación Productiva; 3R: regla de las tres "R" en investigación con animales, reemplazo, refinamiento, reducción, y EFC: Ensayos de Farmacología Clínica. Nota: la representación no implica prelación normativa. 
bases de datos Medline, Lilacs, Bioética OPS/ OMS y PubMed. En estas bases, la búsqueda de artículos sobre bioética, y en particular bioética y ciencia, se efectuó utilizando las palabras clave: "bioética", "ciencia", "bioethics", "science" y "Argentina". Las búsquedas en las bases mencionadas se realizaron entre enero y noviembre de 2008. De cada base consultada se registró el número total de citas indizadas y se investigó la producción bibliográfica más antigua compilada sobre bioética, tanto con filiación argentina en su autoría como con otra filiación. Posteriormente, para el análisis de contenido se seleccionaron los doce últimos trabajos indizados a noviembre de 2008 que cumplieron con los siguientes criterios de inclusión: tratar temas relacionados con bioética y tener al menos un autor con filiación en Argentina. Para la depuración de la selección se consideraron redundantes aquellos trabajos indizados en más de una base y sólo se tuvieron en cuenta una vez. Sobre los documentos seleccionados, se caracterizó año y clase de publicación — contribución en revista especializada, libro, otras. Sobre las contribuciones en revistas se analizó: revista, idioma y ámbito de publicación, contenido y tipo de trabajo. El tipo de trabajo fue clasificado como: i) trabajo original, ii) artículo de reflexión, iii) carta del lector o comentario editorial, iv) documentos sobre comités de ética clínica (CEC) y v) reporte sobre situación de la bioética en el país.

Las bases de datos consultadas se encuentran disponibles en español, ingles y portugués e indizan en su conjunto bibliografía médica, biomédica y de ciencias de la salud, compilando la base de datos Bioética OPS/OMS, en particular, aquella bibliografía referida a bioética, ética médica y salud pública. En cuanto a la cobertura geográfica, Lilacs reúne las producciones provenientes de América del Sur y del Caribe, y las otras bases compilan además la originada en el resto del mundo.

\section{Resultados}

Normas de alcance nacional relacionadas con la investigación cientifica en ciencias de la vida y presencia de elementos de bioética

Se han encontrado normas de alcance nacional vigentes en Argentina, con distinta prelación jurídica - leyes, disposiciones, resoluciones-, promulgadas entre 1954 y $2008^{9}$. Asimismo, se hallaron proyectos de ley vinculados a mecenazgo de la investigación científica, a la creación y funcionamiento de comités de ética para la investigación en salud y a ética en el uso de animales con fines experimentales. Estos proyectos de ley están, al período parlamentario 2008, en instancias de trámite ante las cámaras de Diputados o de Senadores de la Nación. En el Diagrama 1 se muestran las normas y proyectos de ley de alcance nacional hallados y la presencia en su contenido de elementos de bioética.

Análisis de los aspectos éticos requeridos para la publicación de trabajos en ciencias de la vida en Argentina

Se analizaron los reglamentos de 21 revistas argentinas relacionadas con las ciencias de la vida, de las cuales 14 pertenecían a las áreas de ciencias de la salud y ciencias biológicas del CAICYT, representado el $44 \%$ de las revistas compiladas en dichas áreas, y de las no circunscritas en CAICYT, cuatro dependían de colectivos científicos/profesionales y tres de universidades nacionales.

La fecha de actualización de los reglamentos correspondió al período 2003-2008 en 11 casos, y a 1993 en un caso; en los nueve casos restantes no consignaba dicha fecha. De las revistas pertenecientes al CAYCIT, el 50\% tenía sus reglamentos actualizados durante el período mencionado de la década de 2000 y el otro $50 \%$ no refería fecha de actualización. De las revistas no incluidas en CAYCIT — pertenecientes a colectivos y/o universidades-, cuatro presentaban reglamentos actualizados durante aquel período de la década de 2000, una en 1993 y dos no referían fecha de actualización.

El 100\% de los reglamentos analizados acepta la publicación de trabajos y/o contenidos con con-

\footnotetext{
${ }^{9}$ Como ejemplo de la aplicación de las regulaciones referidas a ensayos clínicos con seres humanos, entre 1994 y 2007 ANMAT aprobó, en virtud de aquellas, 1892 protocolos de ensayos clínicos y rechazó 100. Datos de Seoane MR. (2007). La investigación clínica y la ANMAT. Disponible en: www.anmat.gov.ar/cursosyConf/15anios SEOANE.pps. Recuperado el 6 de diciembre de 2008.
} 
notaciones de interés en bioética, siendo ellos: estudios experimentales en humanos, en animales y en el medio ambiente, datos de historias clínicas, fotografías e imágenes diagnósticas obtenidas de pacientes humanos, ensayos clínicos y estudios con manipulación genética, y estudios biotecnológicos. Todas las revistas poseen un comité de pares para la evaluación técnica de los trabajos remitidos y en 1/21 de los casos tiene además un comité de evaluación ética.

De los 21 reglamentos, 15 (71\%) contenían recomendaciones sobre ética en la comunicación científica y 6 (29\%) solicitaban al mismo tiempo el cumplimiento de requisitos de ética en la investigación. Las recomendaciones sobre ética en la comunicación consistieron en: solicitud de autorización a editores de otras revistas para presentaciones simultáneas de una parte o la totalidad del trabajo en otra revista, garantía de originalidad de la comunicación, presentación de conformidad por escrito de los coautores, declaración de conflictos de interés y especificación de financiamientos y/o sponsors.

Los requisitos de ética en la investigación, fijados en los seis reglamentos que los contenían, fueron: aprobación de un comité de ética, presentación de consentimiento informado de los sujetos participantes en el estudio, protección de datos de pacientes, cumplimiento de la Declaración de Helsinki, validación de ensayos clínicos con protocolos internacionales y cumplimiento de reglas de ética en experimentación con animales.

Estudio de la producción bibliográfica en bioética con participación en su elaboración de autores con filiación argentina.

A partir de la exploración en las bases de datos Medline, Lilacs, Bioética y PubMed, con el término "bioética" se recuperaron un total de 8.823 documentos publicados entre 1973 y 2007. De ellos, el 1,2\% tuvo en su elaboración participación total o parcial de autor/res con filiación argentina. A su vez, en dichas bases y con el término "bioethics" se recuperaron 29.562 documentos publicados entre 1972 y 2008 , y el $0,34 \%$ presentó la participación mencionada. El trabajo más antiguo compilado, de autoría con filiación argentina, correspondió a un artículo de Sanguinetti de $1988^{10}$, y para autores con otra filiación correspondió a un artículo de Potter de 1972. El número de documentos y período de publicación de las citas indizadas según palabra clave utilizada y base de datos consultada se muestra en la Tabla 1.

Tabla 1. Número de documentos según palabra clave utilizada y base de datos consultada y período de publicación [ ] de las citas indizadas, a noviembre de 2008.

\begin{tabular}{|c|c|c|c|}
\hline $\begin{array}{l}\text { Palabra clave } \\
\text { Base de datos }\end{array}$ & $\begin{array}{l}\text { Bioética } \\
\text { Bioethics }\end{array}$ & $\begin{array}{l}\text { Bioética, Argentina } \\
\text { Bioethics, Argentina }\end{array}$ & $\begin{array}{l}\text { Bioética, ciencia, } \\
\text { Argentina } \\
\text { Bioethics, science, } \\
\text { Argentina }\end{array}$ \\
\hline LILACS $^{1}$ & $\begin{array}{l}2.214 /[2008-1981] \\
2.114 /[2008-1981]\end{array}$ & $\begin{array}{l}40 /[2007-1988] \\
39 /[2007-1988]\end{array}$ & $\begin{array}{l}8 /[2004-1994] \\
4 /[2004-1998]\end{array}$ \\
\hline MEDLINE $^{1}$ & $\begin{array}{l}5.642 /[2008-1973] \\
3.345 /[2008-1972]\end{array}$ & $\begin{array}{l}17 /[2007-1990] \\
11 /[2007-1990]\end{array}$ & $\begin{array}{l}2 /[1993-1990] \\
4 /[2007-1991]\end{array}$ \\
\hline PUBMED $^{2}$ & $\begin{array}{c}160 /[2008-1990] \\
17.354 /[2008-1972]\end{array}$ & $\begin{array}{c}9 /[2007-1996] \\
46 /[2008-1990]\end{array}$ & $\begin{array}{c}0 \\
15 /[2008-1990]\end{array}$ \\
\hline BIOÉTICA $^{1}$ & $\begin{array}{c}807 /[2003-1993] \\
149 /[2003-1994]\end{array}$ & $\begin{array}{c}41 /[1994-1990] \\
5 /[1999-1996]\end{array}$ & $\begin{array}{c}2 /[1996-1990] \\
0\end{array}$ \\
\hline TOTAL & $\begin{array}{c}8.823 /[2008-1973] \\
29.562 /[2008-1972]\end{array}$ & $\begin{array}{l}107 /[2007-1988] \\
101 /[2008-1988]\end{array}$ & $\begin{array}{l}12 /[2004-1990] \\
23 /[2008-1990]\end{array}$ \\
\hline
\end{tabular}

${ }^{1}$ Fecha de acceso para la última búsqueda 28/10/2008. ${ }^{2}$ Fecha de acceso para la última búsqueda 27/10/2008.

En el análisis de contenido del total de documentos estudiados $(\mathrm{N}=48), 40$ correspondieron a la clase de contribución en revista, cuatro a libros y en cuatro casos no se pudo identificar su clase. En relación con el idioma de la publicación del documento, 34 estaban publicados en español, 13 en inglés y uno en portugués. Todos los documentos seleccionados de la base de datos Bioética OPS/ OMS estaban en español, al igual que los de Lilacs, salvo uno publicado en portugués. Entre aquellos documentos seleccionados de PubMed y Medline se encontraban los 13 publicados en inglés y 11 publicados en español.

En cuanto al período de publicación de las producciones exploradas, 27 fueron publicadas durante la década del 2000 y 21 durante la década de 1990, correspondiendo el documento más antiguo de los seleccionados para el análisis de contenido al año 1990, y los más recientes a 2007. La base de datos Bioética OPS/OMS presentó, al momento de su revisión, la indización de 13/21 de los documentos publicados durante la década

${ }^{10}$ Sanguinetti FA. (1988). Responsabilidad ética y jurídica del cirujano. Revista Argentina de Cirugía. 55(n.esp): 5-55. 
de 1990 y de $1 / 27$ de los publicados en la década del 2000. Las bases de datos Medline, PubMed y Lilacs presentaron en su conjunto la indización de $8 / 21$ y $26 / 27$ de los documentos publicados durante la década de 1990 y 2000 respectivamente, al momento de su exploración.

La revista con mayor número de documentos publicados fue Vertex, siguiéndole en orden de abundancia Bioethics, el Boletín de la Oficina Panamericana de la Salud y Medicina (Buenos Aires). En la Tabla 2 se muestra la distribución, según revista de publicación, de las 40 contribuciones analizadas.

Tabla 2. Distribución según revista de publicación de 40 contribuciones sobre bioética de autoría total o parcial con filiación argentina. Datos expresados en frecuencia absoluta y relativa. Producciones indizadas a noviembre de 2008 en bases de datos internacionales ${ }^{1}$.

\begin{tabular}{|l|c|c|}
\hline Revista o clase de publicación & $\begin{array}{c}\text { Frecuencia } \\
\text { absoluta (n) }\end{array}$ & $\begin{array}{c}\text { Frecuencia } \\
\text { relativa (\%) }\end{array}$ \\
\hline Vertex & 6 & 15 \\
Bioethics & 3 & 7 \\
Boletín Oficina Sanitaria Panamericana & 3 & 7 \\
Medicina (Buenos Aires) & 3 & 7 \\
Journal of Medical Philosophy & 2 & 4 \\
Law Human Genome Reviews & 2 & 4 \\
Quiron & 2 & 4 \\
Medicina y Sociedad & 2 & 4 \\
Acta Bioethica & 2 & 4 \\
American Journal of Bioethics & 2 & 4 \\
Bioética & 1 & 2 \\
CM Publicaciones Médicas & 1 & 2 \\
Revista Asociación Médica Argentina & 1 & 2 \\
Revista Argentina de Cirugia & 1 & 2 \\
Perspectivas Bioéticas Americana & 1 & 2 \\
Derecho (Bs. As.) & 1 & 2 \\
Cuadernos Programa Regional de Bioética & 1 & 2 \\
Cuadernos de Bioética (Espana) & 1 & 2 \\
IUBMB Life2 & 1 & 2 \\
Developing World Bioethics & 1 & 2 \\
Eubios Journal Asian Bioeth. & 1 & 2 \\
Journal International of Bioethique & 1 & 2 \\
Journal of Kennedy Institute of Ethics & 1 & 2 \\
\hline Bases de datos: PubMed, Medline, Lilacs, Bioetica OPSTOMS,
\end{tabular}

${ }^{2}$ IUBMB Life: International Union of Biochemistry and Molecular Biology.

El 50\% de las contribuciones analizadas correspondió al tipo de artículo de reflexión, seguido en orden de frecuencia de aparición por trabajo original (27\%), documento sobre CEC (9\%), reporte de situación de la bioética en el país (9\%) y carta del lector/comentario editorial (6\%). El contenido abordado con más frecuencia fue funcionamiento de los comités de ética clínica $(50 \%)$, seguido por temas de interés bioético (25\%) — tales como la información al paciente, la confidencialidad, el aborto y la eutanasia-, ética e investigación (13\%), formación en bioética (10\%) y biojurídica (2\%).

\section{Discusión}

La bondad y el valor de la ciencia, como praxis social, radica tanto en la verdad que ofrece como en su perfección técnica, en el acuerdo con las exigencias éticas del ser humano y en la perfección que adquiera, y en la honradez intelectual del investigador que la lleve a cabo $(2,15)$. En ayuda del científico se presenta la bioética, disciplina práctica que sirve para orientar la acción humana en un sentido racional, analizar los hechos desde una perspectiva ética y tomar decisiones con inclusión de valores.

La participación de los países de América Latina en las investigaciones biomédicas está creciendo exponencialmente y, aunque en forma dispar, las naciones de la región han establecido distintos marcos reglamentarios para asegurar la protección a los seres humanos que son objeto de experimentación(16). En dichos marcos, las leyes conforman un hecho social, ya que son producidas por un conjunto de personas e intentan que la sociedad sea justa, garantizando los derechos de los ciudadanos. Desde una perspectiva amplia, cabe considerar que la coincidencia entre lo ético y lo legal puede suponer una situación ideal, distinguiéndose entre aquello que es legal — de acuerdo con la ley positiva- de aquello que es legítimo o lícito - conforme a la ética $(2,9)$.

Las normas de alcance nacional analizadas en este estudio abarcan, en referencia a la esfera de las ciencias de la vida, algunos de los campos de la investigación en seres humanos — principalmente investigación clínica-, en el ambiente y en el uso de animales de experimentación. En lo referido a investigación biomédica destacan las resoluciones y disposiciones de ANMAT sobre estándares éticos de los ensayos clínicos en humanos y, de verse substanciado, el proyecto de ley sobre comités de ética en investigación en salud ${ }^{11}$.

En la mayoría de las normas analizadas se constató la presencia de elementos de la bioética, tales como la inclusión de la Declaración de Helsinki,

\footnotetext{
${ }^{11}$ Información, consideraciones y análisis detallado sobre comités de ética en investigación en Argentina se encuentra en Outomuro D. Reflexiones sobre el estado actual de la ética en investigación en Argentina. Acta Bioethica 2004; 10(1): 81-94.
} 
el Código de Nürenberg, el consentimiento informado y la confidencialidad, en lo que hace a normas sobre investigación en humanos. También, los principios de precaución y prevención relacionados con la investigación ambiental y los principios de reducción, reemplazo y refinamiento en la experimentación con animales. Dicha presencia otorga un valor de legitimidad a las mismas.

La comunicación en el ámbito de la ciencia es fundamental para compartir saberes y ayudar al avance en el conocimiento. Según un sondeo de Outomuro(8), eran escasas las investigaciones médicas publicadas en Argentina que manifestaban explícitamente respetar los lineamientos de la ética en investigación. En el presente trabajo se constató que sobre 21 reglamentos de revistas argentinas relacionadas con ciencias de la vida, todos aceptaban la presentación de trabajos y/o contenidos con connotaciones éticas y que, si bien dichas revistas poseen un comité evaluador técnico, sólo una tiene además un comité específico para la valoración ética de los trabajos. Asimismo, se observó que la mitad de los reglamentos estaban actualizados al período 2003-2007, uno al año 1993 y un porcentaje considerable (43\%) no refería fecha de actualización. El grado de actualización de los reglamentos de publicación y la posibilidad de evaluar, por parte de un comité especializado en ética, los trabajos enviados a las revistas, cobra especial relevancia en el campo de las ciencias de la vida.

En los reglamentos analizados se ha comprobado la presencia de requerimientos sobre ética en la comunicación científica ( $71 \%$ de los casos) y, en una menor proporción (29\%), de requisitos de ética en la investigación científica. La inclusión de lineamientos de ética en investigación en los reglamentos de publicación comportaría un aporte orientador significativo para los investigadores, colaborando en el conocimiento y difusión de los fundamentos y requerimientos de ésta índole en la ciencia. Cabe señalar al respecto que, según la Declaración de Helsinki(17), no sólo los autores sino también los redactores y los editores se consideran responsables, desde el punto de vista ético, de la publicación de los resultados de una investigación. En este sentido las observaciones emergentes del presente trabajo, sumadas a las referidas de Outomuro(8), indicarían que en Argentina es necesario fomentar la consideración de los aspectos éticos en las bases reglamentarias de las revistas vinculadas a las ciencias de la vida.

La bibliometría se ha extendido como técnica metodológica imprescindible para la evaluación de la producción científica, siendo además una herramienta fundamental para conocer el presente y reconstruir la historia de la investigación en diversas parcelas de la ciencia(18). Por medio del sondeo bibliográfico realizado se observó una diferencia de 16 años entre las fechas de publicación de las primeras producciones compiladas en las bases consultadas; para autores argentinos, correspondió a Sanguinetti, año 1988, y para otras filiaciones correspondió a Potter, año 1972. Existe cierta coincidencia temporal entre aquellas fechas y las consideradas para la aparición de la bioética como disciplina formal en el ámbito internacional y en Argentina. Los primeros años de la década de 1970 son los apuntados como iniciales para el ámbito internacional(19) —en relación principalmente con las aportaciones de Potter-, y 1982 para Argentina(7) —en relación con la contribución de J.A. Mainetti.

La mayoría de los trabajos analizados correspondieron a la clase de contribución en revista (92\%) y, dentro de ella, al tipo de artículo de reflexión. En los contenidos hubo un predominio de tratamiento de temas en torno al desempeño de comités de ética hospitalaria, confidencialidad, aborto y eutanasia, dándose a su vez una menor presencia de contenidos sobre ética e investigación.

Si bien en lo individual la revista con mayor número de documentos publicados sobre bioética fue Vertex - revista de edición nacional especializada en psiquiatría-, se vio que, en su conjunto, el $57 \%(n=40)$ de las producciones han sido publicadas en revistas de editoriales internacionales, tales como Bioethics, Boletín de la Oficina Panamericana de la Salud, Journal of Medical Philosophy, Law Human Genome Reviews, Acta Bioethica y American Journal of Bioethics, entre otras. Se destaca que varias de las revistas internacionales en las que se hallaron los trabajos son del ámbi- 
to iberoamericano. Ello, sumado a la posibilidad de publicación a través de editoriales nacionales, podría explicar el predominio del español como idioma de publicación principal de las producciones.

La producción en bioética desde Argentina parecería estar levemente incrementándose. Tomando el período de publicación de los trabajos seleccionados para el análisis de contenido, se dio que $56 \%$ de los mismos correspondió a documentos publicados entre 2000 y 2007, mientras que un $44 \%$ se publicó durante la década de 1990 . Esta observación se realiza en línea de hipótesis, sobre la base de representatividad que supone el análisis de un tamaño de muestra de $45 \%$ del total de citas recuperadas con las palabras clave "bioética" y "Argentina" - $\mathrm{N}=107$ - en las bases consultadas.

No obstante las observaciones antes referidas, la presencia en el plano internacional de la producción en bioética de autores con filiación argentina aún resultaría mínima en comparación con la producción de otras filiaciones, en particular de la anglosajona. Según un estudio de Borry et al.(20), los autores anglosajones comportan el $80 \%$ de la autoría de las contribuciones en el campo de la bioética, y la escasa representación de autores de otras filiaciones se debe a barreras lingüísticas, a la falta de recursos para desarrollar investigaciones en la disciplina bioética, a la dificultad de acceso a revistas internacionales y/o a tendencias editoriales. Estas situaciones podrían estar también influyendo sobre la producción argentina en la disciplina y su representación en el escenario de las producciones bibliográficas en el ámbito internacional.
La bioética trata cada vez más de amparar los dilemas éticos relacionados con la atención sanitaria y la investigación con seres humanos, y se ocupa, a su vez, de las relaciones que surgen con la familia, la comunidad científica y social, con otros seres vivos y con todo el ecosistema(21). Sobre la base de los resultados obtenidos a través del presente trabajo se vislumbran situaciones que pueden contribuir a una consolidación de la bioética en relación con las ciencias de vida en Argentina, tales como la incorporación de elementos de la bioética al ámbito legal de alcance nacional, y la aportación desde 1988 de autores argentinos a la producción bibliográfica en la disciplina. Esta última situación, junto con la incorporación de lineamientos de ética en la investigación científica en revistas argentinas relacionadas con dichas ciencias, se considera, según lo evidenciado, como campo a fomentar en el contexto de consolidación disciplinar referido.

\section{Agradecimientos}

Parte de la información del presente trabajo proviene de la Tesis de final de Máster Oficial en Bioética (Universidad Católica de Valencia, España) de Paula Sánchez Thevenet, realizada bajo la dirección del Dr. José Hernández Yago, presidente de la Sociedad Valenciana de Bioética (España), jefe del Laboratorio de Organización Celular del Centro de Investigación Príncipe Felipe (Valencia, España) y profesor Titular del Departamento de Biotecnología de la Universidad Politécnica de Valencia (Espańa), a quien los autores agradecen su ayuda técnica y autorización para esta publicación. 


\section{Referencias}

1. De Jesús Sierra Cuartas CJ. Bioética e historia de la ciencia y la tecnología: un portal atípico para el abordaje temprano de la bioética. Bioética Revista Latinoamericana 2009; 2(2): 1-31.

2. Franco L. Ética de la investigación, ética del investigador. En: Sociedad de Bioética Valenciana (comp.) Cuestiones de bioética. Valencia: Antolín Martínez Imp.; 1996: 96-106.

3. Ferrer Santos U. Ciencia, ética y bioética. Cuadernos de Bioética 2003; 2-3, 195-207.

4. Herranz J. La humanidad ante una encrucijada: derecho y biología. Cuadernos de Bioética 2001; 12 (46): 329-344.

5. De Oliveira Rivero CR, Pavone Zoboli EL. Pobreza, bioética e investigación. Revista Latino-Americana de Enfermagem 2007; 15 (n. spe): 843-849.

6. Holm S, Williams-Jones B. Global bioethics-myth or reality?. BMC Medical Ethics 2006; 7: 1-10.

7. Macklin R, Luna F. Bioethics in Argentina: a country report. Bioethics 1996; 10(2): 140-153.

8. Outomuro D. Reflexiones sobre el estado actual de la ética en investigación en Argentina. Acta Bioethica 2004; 10(1): 81-94.

9. Outomuro D. Algunas observaciones sobre el estado actual de la bioética en Argentina. Acta Bioethica 2003; 10(2): 229-238.

10. EULABOR. European and Latin American Systems of Ethics Regulation of Biomedical Research. SAS6-CT-2005-51786: Descripción de los sistemas de regulación ética nacionales, Argentina; 2005.

11. Miniyersky N, Flah LR. Consentimiento informado de niñas, niños y adolescentes. Derecho personalisimos. Influencia del reconocimiento de la capacidad progresiva en la protección de la salud. En: Actas VI Congreso Mundial de Bioética (Gijón) 2009; 280-281.

12. Sánchez Thevenet P. Bioética e investigación en Argentina: exploración inicial de la vulnerabilidad del sujeto de investigación. En: Actas VI Congreso Mundial de Bioética (Gijón) 2009; 226-228.

13. Basualdo JA, Minvielle MC, Grenóvero MS. Nociones básicas de la metodología de la investigación en ciencias de la salud, Tomo 2. $1^{\text {a }}$ ed. Argentina (La Plata); 2004.

14. UNESCO. Proposed international standart nomenclature for fields of science and technology. Francia (Paris): UNESCO/NS/ROU/257; 1988.

15. Bellver Capella V. Bioética, ciencia y medios de comunicación. En: Bellver Capella, V (comp.) Por una bioética razonable. Granada: Editorial Comares; 2005: 10-64.

16. Litewka S, Goodman K, Braunschweiger P. El programa CITI: una alternativa para la capacitación en ética de la investigación en América Latina. Acta Bioethica 2008; 14(1): 54-60.

17. World Medical Association. Declaration of Helsinki: ethical principles for medical research involving human subjects; 2008.

18. Delgado López-Cózar E, Torres-Salinas D, Jiménez-Contreras E, Ruiz-Pérez R. Análisis bibliométrico y de redes sociales aplicado a las tesis defendidas en España (1976-2002): temas, escuelas científicas y redes académicas. Revista Española de Documentación Científica 2006; 29(4): 1-36.

19. Suazo Báez MA. La bioética, su génesis y sus alcances. Bioética Latinoamericana 2008; 1: 1-11.

20. Borry P, Schotsmans P, Dierickx K. How intenational is bioethics? A quantitative retrospective study. BMC Medical Ethics 2006; 7: 1-6.

21. Tomás y Garrido GM. (comp.) Manual de Bioética. 1a ed. España (Barcelona): Editorial Ariel Ciencia; 2001.

Recibido: 24 de agosto de 2009

Aceptado: 15 de marzo de 2010 